\title{
Taxonomic Diversity of Lianas in Tropical Forests of Northern Eastern Ghats of Andhra Pradesh, India
}

\author{
Maradana TARAKESWARA NAIDU, Owk ANIEL KUMAR, Malleboyina \\ VENKAIAH
}

\begin{abstract}
Department of Botany,Andhra University,Visakhapatanam-530003,India; tarakeswaranaidu@gmail.com; oak.aniel@gmail.com; venkaiahbotany@rediffmail.com
\end{abstract}

\begin{abstract}
Lianas are important in forest ecosystem and strongly influence the forest dynamics and diversity. Lianas are common in the tropical moist deciduous and rain forests, which are competing with other forest trees. Little information is known on the habitat specialization in tropical lianas diversity and the root causes for variation among forests in liana species composition. A total of 170 liana species ( $\geq 1.5 \mathrm{~cm}$ girth at breast height) representing 109 genera and 43 families were reported in $5 \times 5$ m quadrate samples along with their climbing modes in the tropical forests of northern Eastern Ghats of Andhra Pradesh, India. A total of 210 grids were sampled in study area and reported that Convolvulaceae was the dominant family with 23 species followed by Papilionaceae, 22 species and Asclepiadaceae, 19 species and Ipomoea was the largest genera. Woody lianas were dominated by 128 species and these are classified into six climbing modes consisting in stem climbers $(53.5 \%)$ that were the most predominant followed by stragglersunarmed (14.7\%), stragglers armed and tendril climbers (13.5\% each), root climbers (2.9\%) and hook climbers (1.8\%). The most dominant liana species in the northern Eastern Ghats were Acacia sinuata and Baubinia vablii. The results of this investigation suggests that better management and protection is an important for in situ conservation of liana diversity and involving local people is emphasized.
\end{abstract}

Keywords: climbing modes, conservation, diversity, Eastern Ghats, lianas

\section{Introduction}

Lianas are long-stemmed woody vines, which are fixed in the soil at ground level and depend on the physical support of other plants to reach the forest canopy (Araujo and Alves, 2010; Schnitzer and Bongers, 2002). These are prominent features of most tropical forests, where their leaves can constitute a large amount of the total area of the entire forest community (Putz and Mooney, 1991). Lianas make use of much greater ecological consequence than their size suggests and represent less than $5.0 \%$ of tropical forest biomass but up to $40 \%$ of leaf productivity (Hegarty and Caballe, 1991; Heidjen and Phillips, 2008). They struggle strongly with trees, very much reducing tree growth, tree reproduction and greatly increasing tree mortality (Wright et al., 2005; Schnitzer and Carson, 2010; Ingwell et al., 2010) and altering the course of regeneration in forests (Schnitzer $e t$ al., 2000). The density of lianas was greater than ever before and significantly increased their diversity during the last two decades of the twentieth century (Phillips et al., 2002).

The ecological importance of lianas is well documented, since they are of fundamental importance in the functioning of ecosystems as competing with trees either directly or indirectly. They act as key ecological components of whole forest in transpiration, carbon sequestration and forest regeneration (Schnitzer and Bongers, 2002). Lianas play a significant ecological role in different patterns of pollination, dispersal and phenological systems, provide several resources, and play vital roles in the protection of biological diversity (Reddy and Parthasarathy, 2006). The wealth and species diversity of lianas also depend upon a number of abiotic factors, including total occurrence of rainfall with seasonal variations, soil fertility and disturbances (Schnitzer, 2005). The main causes of the disturbance of lianas are the tree fallings not only important for sustaining the liana species but also maintain diversity that leads to increased development of lianas (Yuan et al., 2009; Reddy and Parthasarathy, 2003). The development of highest frequency of lianas is mainly because of declining the rainfall but several factors that are known to favour them are the increase anthropogenic impacts (Londre and Schnitzer, 2006). Lianas act as an indicator species response to increase $\mathrm{CO}_{2}$ concentrations and benefit from other anthropogenic 
disturbances (Zotz, 2006). A few studies on lianas have been carried out in the Eastern Ghats forests, India (Muthumperumal and Parthasarathy, 2009; Reddy and Parthasarathy, 2003; Parthasarathy et al., 2004). However, most of the studies were not exclusively on lianas as they involve general botanical surveys with reference to herbaceous, shrub and tree flora. Thus, main objective of present study was to inventory the lianas diversity and ecological findings in the northern Eastern Ghats forests of Andhra Pradesh, India.

\section{Materials and methods}

The Eastern Ghats are a long chain of broken hills and elevated plateaus and one of the nine floristic regions in India, running along the east coast of India in the states of Odisha, Andhra Pradesh, Tamil Nadu and Karnataka and lies between Mahanadhi and Vaigai rivers. The present study was carried out in northern Eastern Ghats of Andhra Pradesh, which lies between latitudes of $16^{\circ} 15^{\prime}$ and $19^{\circ} 12^{\prime}$ $\mathrm{N}$ and eastern longitudes of $80^{\circ} 50^{\prime}$ and $84^{\circ} 47^{\prime} \mathrm{E}$ runs through five districts, namely Srikakulam, Vizianagaram, Visakhapatnam, East Godavari and West Godavari. The highest elevation measures about $1615 \mathrm{~m}$ above the msl in this region. Geological formation of the region consists chiefly in Charnokites and Kondalites and varied metamorphic rocks. Soils of northern Eastern Ghats is loamy, black, lateritic and alluvial. Lateritic soils are the common type along the deciduous forests of the area. The climate of the district is characterized by uncomfortably hot during summer and pleasantly cold during winter. There are three distinct seasons in a year; winter (November to February), summer (March to June) and rainy season (July to October). The maximum temperature ranges from $28^{\circ}-$ $46.2^{\circ} \mathrm{C}$ and minimum temperature ranges between $12.9^{\circ}-$ $27^{\circ} \mathrm{C}$. The maximum rainfall is $1300 \mathrm{~mm}$ per annum in south-west monsoon period. At all the plots, a similar pattern of temperature and rainfall prevails throughout the year. The relative humidity varied between $70-88 \%$. The forests in northern Eastern Ghats are broadly classified into Tropical Semi-evergreen, Tropical Moist Deciduous, Tropical Dry Deciduous, Tropical Thorny-Scrub vegetation and Tropical Dry Evergreen forest types (Champion and Seth, 1968).

The field work was carried out in a total 210 grids in the forests of northern Eastern Ghats of Andhra Pradesh for enumeration of lianas. The entire stretch of northern Eastern Ghats of Andhra Pradesh was divided into $6.25 \times 6.25 \mathrm{~km}$ grids and within each grid a $0.5 \mathrm{ha}$ transect $(5 \mathrm{~m} \times 1 \mathrm{~km})$ was laid. Depending on the shape of the forest stand, these transects were divided into $5 \mathrm{x}$ $200 \mathrm{~m}$ sub transects. All lianas $-1.5 \mathrm{~cm}$ gbh $(5 \mathrm{gbh}$, girth at breast height) were enumerated in the whole transects, and those of herbaceous vines of $<1.5 \mathrm{~cm}$ gbh only in the beginning and end of the transects. The collected specimens were identified with the help of floras (Gamble and Fischer, 1915-1935; Rao and Kumari, 2002 - 2008). The voucher specimens were deposited in the Botany Department Herbarium (BDH), Department of Botany, Andhra University, Visakhapatnam.

\section{Results and discussion}

The study area contained a total of 170 liana species representing 109 genera and 43 families, recorded from northern Eastern Ghats of forests in the total of 210 grids (Tab. 1). Muthumperumal and Parthasarathy (2009) enumerated 175 angiosperm climbing plants in 150 grids of southern Eastern Ghats; 60 liana species found in Maruthamalai hills of southern Western Ghats (Sarvalingam and Rajendran, 2012); 93 climbing plant species reported in land Atlantic forest, northern Brazil (Araujo and Alves, 2010); the total number of climbers that are found in Puerto Rico and the Virgin Islands amounts to 386 (AcevedoRodriguez, 2005). The present study identified a genus and species ratio of $1: 1.55$. Out of 170 species, only one species was Gymnosperm i.e. Gnetum ula and 169 species consist of 108 genera and 42 families were angiosperms. Among the angiosperms, there were 154 species representing 36 families of dicotyledons and 15 species belonging to 6 monocotyledon families.

The most specious families investigated in the present study include Convolvulaceae (23 species), Papilionaceae (22 species), Asclepiadaceae (19 species), Cucurbitaceae ( 9 species), Dioscoreaceae, Menispermacae and Vitaceae (8 species each) etc., while in southern Eastern Ghats Asclepidaceae, Convolvulaceae, Papilionaceae, Apocynaceae, Vitaceae and Menispermaceae formed the most dominant familes (Muthumperumal and Parthasarathy, 2009). Papilionaceae, Cucurbitaceae, Convolvulaceae and Asclepiadaceae formed the common liana families in Maruthamalia hills of Western Ghats (Sarvalingam and Rajendran, 2012). Families such as Smilacaceae, Menispermaceae, Passifloraceae, Cucurbitaceae and Convolvulaceae are entirely dominated by species with a climbing habit (Araujo and Alves, 2010). According to Gentry (1991) New World families, with the highest diversity of climbing plants, are Apocynaceae (esp. Asclepiadoideae), Convolvulaceae and Papilionaceae. The dominant genera in the present study were Ipomoea (9 species), Dioscorea (8 species), Argyreia and Merremia (5 species each). Muthumperumal and Parthasarathy (2009) recorded that Jasminum, Acacia, Argyreia and Capparis were the most abundant genera in southern Eastren Ghats, while in Maruthamalia hills of Western Ghats Ipomoea and Rhynchosia were the dominant genera (Sarvalingam and Rajendran, 2012).

The most dominant liana species in the study are Acacia sinuata (Mimosaceae), Baubinia vablii (Caesalpiniaceae), Calycapteris floribunda (Combretaceae), Combretum albidum (Combretaceae), Dioscorea pentalhylla (Dioscoreaceae), Tinospora cordifolia (Menispermaceae) Schefflera stellata (Araliaceae) and Ziziphus oenoplia (Rhamnacea). The rare species were Anodendron paniculatum (Apocynaceae), Aristolochia tagala (Aristolochiaceae), Clematis smilacifolia (Ranunculaceae), Entada pursaetha (Mimosaceae), Gnetum ula (Gnetaceae), Leptadenia reticulata (Aslepiadaceae), Uncaria sessilifructus (Rubiaceae) and Vanilla wightiana (Orchidaceae).

The enumerated lianas classified into woody vines (128 species) and herbaceous vines (42). The mechanisms of the climbers to get attached to their host plants play a major role 
61

in their distribution (Nabe-Nielsen, 2001). In this study, six major mechanisms of climbing systems were recognized: stem twiners (53.5\%) followed by stragglers-unarmed (14.7\%), stragglers-armed $(13.5 \%)$, tendril climbers (13.5\%), root climbers $(2.9 \%)$ and hook climbers (1.8\%). Several authors have reported that stem twiners were most common in the different tropical forests (Ghollasimood et al., 2012; Parthasarathy et al., 2004; Kuzee and Bongers, 2005). Burnham (2004) suggested that the basic information about lianas regarding the location of the species is hard to compile because vouchering, reporting and sampling have been standardized for comparable tree communities.

Tab.1. List of liana species in northern Eastern Ghats of Andhra Pradesh

\begin{tabular}{|c|c|c|c|c|}
\hline No. & Plant species & Family & Category & $\begin{array}{c}\text { Climbing } \\
\text { mode }\end{array}$ \\
\hline 1 & Abrus precatorius $\mathrm{L}$. & Papilionaceae & WV & ST \\
\hline 2 & Acacia caesia (L.) Willd. & Mimosaceae & WV & Str-A \\
\hline 3 & Acacia pennata (L.) Willd. & Mimosaceae & WV & Str-A \\
\hline 4 & Acacia sinuata (Lour.) Merr. & Mimosaceae & WV & Str-A \\
\hline 5 & Acacia torta (Roxb.) Craib. & Mimosaceae & WV & Str-A \\
\hline 6 & Aganosma dichotoma (Roth) K. Schum. & Apocynaceae & WV & ST \\
\hline 7 & Ampelocissus latifolia (Roxb) Planch. & Vitaceae & WV & $\mathrm{TC}$ \\
\hline 8 & Ampelocissus tomentosa (Roth) Planch. & Vitaceae & WV & $\mathrm{TC}$ \\
\hline 9 & Anamitra cocculus (L.) Wt. \& Arn. & Menispermaceae & WV & ST \\
\hline 10 & Anodendron paniculatum (Roxb.) DC. & Apocynaceae & WV & ST \\
\hline 11 & Argyreia arakuensis $\mathrm{Bal}$. & Convolvulaceae & WV & ST \\
\hline 12 & Argyreia daltoni $\mathrm{Cl}$. & Convolvulaceae & WV & ST \\
\hline 13 & Argyreia involucrata $\mathrm{Cl}$. & Convolvulaceae & WV & ST \\
\hline 14 & Argyreia nervosa (Burm.f.) Boj. & Convolvulaceae & WV & ST \\
\hline 15 & Argyreia roxburghii Choisy & Convolvulaceae & WV & ST \\
\hline 16 & Aristalochia indica $\mathrm{L}$. & Aristolochiaceae & HV & ST \\
\hline 17 & Aristolochia tagala Cham. & Aristolochiaceae & WV & ST \\
\hline 18 & Asparagus racemosus Willd. & Liliaceae & HV & Str-A \\
\hline 19 & Atylosia albicans (Wt. \& Arn.) Benth. & Papilionaceae & WV & ST \\
\hline 20 & Atylosia scaraboides (L.)Benth. & Papilionaceae & WV & ST \\
\hline 21 & Atylosia volubilis (Blanco) Gamble & Papilionaceae & WV & ST \\
\hline 22 & Baubinia vablii Wt. \& Arn. & Caesalpiniaceae & WV & Str-UA \\
\hline 23 & Bridelia stipularis (L.) Bl. & Euphorbiaceae & WV & Str-UA \\
\hline 24 & Butea superba Roxb. & Papilionaceae & WV & Str-UA \\
\hline 25 & Caesalpinia decapetala (Roth) Alston & Caesalpiniaceae & WV & Str-A \\
\hline 26 & Caesalpinia digyna Rottl. & Caesalpiniaceae & WV & Str-A \\
\hline 27 & Calycopteris floribunda Lam. & Combretaceae & WV & ST \\
\hline 28 & Canavalia gladiata (Jacq.) DC. & Papilionaceae & WV & ST \\
\hline 29 & Canavalia virosa (Roxb.) Wt. \& Arn. & Papilionaceae & WV & ST \\
\hline 30 & Capparis divaricata Lam. & Capparaceae & WV & Str-A \\
\hline 31 & Capparis zeylanica L. & Capparaceae & WV & Str-A \\
\hline 32 & Cardiospermum helicacabum $\mathrm{L}$. & Sapindaceae & $\mathrm{HV}$ & $\mathrm{TC}$ \\
\hline 33 & Carissa carandas $\mathrm{L}$. & Apocynaceae & WV & Str-A \\
\hline 34 & Carissa inermis Vahl. & Apocynaceae & WV & Str-A \\
\hline 35 & Cassytha filiformis $\mathrm{L}$. & Lauraceae & HV & ST \\
\hline 36 & Cayratia auriculata (Roxb.) Gamble & Vitaceae & WV & TC \\
\hline 37 & Cayratia pedata (Lam.) Gangep. & Vitaceae & WV & $\mathrm{TC}$ \\
\hline 38 & Cayratia trifolia (L.) Domin. & Vitaceae & WV & TC \\
\hline 39 & Celastrus paniculatus Willd. & Celastraceae & WV & Str-UA \\
\hline 40 & Ceropegia bulbosa Roxb. & Asclepiadaceae & $\mathrm{HV}$ & ST \\
\hline 41 & Cissampelos pareira $\mathrm{L}$. & Menispermaceae & HV & ST \\
\hline 42 & Cissus quadrangularis $\mathrm{L}$. & Vitaceae & WV & $\mathrm{TC}$ \\
\hline
\end{tabular}




\begin{tabular}{|c|c|c|c|c|}
\hline 43 & Cissus repanda Vahl. & Vitaceae & $\mathrm{WV}$ & TC \\
\hline 44 & Cissus vitiginea $\mathrm{L}$. & Vitaceae & WV & TC \\
\hline 45 & Clematis gouriana DC & Rananculaceae & WV & Str-UA \\
\hline 46 & Clematis roylei Rehder & Rananculaceae & WV & Str-UA \\
\hline 47 & Clematis smilacifolia Wall. & Rananculaceae & WV & Str-UA \\
\hline 48 & Clitoria ternatea $\mathrm{L}$. & Papilionaceae & WV & ST \\
\hline 49 & Coccinia grandis (L.) Voigt & Cucurbitaceae & WV & TC \\
\hline 50 & Cocculus hirsutus (L.) Diels & Menispermaceae & WV & ST \\
\hline 51 & Combretum albidum G. Don & Combretaceae & WV & ST \\
\hline 52 & Combretum roxburghii Spreng. & Combretaceae & WV & ST \\
\hline 53 & Cryptolepis buchanani Roem. \& Schult. & Asclepiadaceae & WV & ST \\
\hline 54 & Cryptolepis elegans Don & Asclepiadaceae & WV & ST \\
\hline 55 & Cynanchum callialatum $\mathrm{Wt}$. & Asclepiadaceae & WV & ST \\
\hline 56 & Dalbergia volubilis Roxb. & Papilionaceae & WV & Str-UA \\
\hline 57 & Derris scandens (Roxb.) Benth. & Papilionaceae & WV & ST \\
\hline 58 & Dioscorea anguina Roxb. & Dioscoreaceae & HV & ST \\
\hline 59 & Dioscorea bulbifera $\mathrm{L}$. & Dioscoreaceae & $\mathrm{HV}$ & ST \\
\hline 60 & Dioscorea glabra auct. & Dioscoreaceae & $\mathrm{HV}$ & ST \\
\hline 61 & Dioscorea hamaltonii Hook.f. & Dioscoreaceae & $\mathrm{HV}$ & ST \\
\hline 62 & Dioscorea hispida Dennst. & Dioscoreaceae & $\mathrm{HV}$ & ST \\
\hline 63 & Dioscorea oppositifolia L. & Dioscoreaceae & $\mathrm{HV}$ & ST \\
\hline 64 & Dioscorea pentaphylla L. & Dioscoreaceae & HV & ST \\
\hline 65 & Dioscorea tomentosa Spreng. & Dioscoreaceae & $\mathrm{HV}$ & ST \\
\hline 66 & Diplocyclos palmatus (L.) Jeffrey & Cucurbitaceae & WV & TC \\
\hline 67 & Dolichos trilobus L. & Papilionaceae & $\mathrm{HV}$ & ST \\
\hline 68 & Embelia ribes Burm.f. & Myrsinaceae & WV & Str-UA \\
\hline 69 & Entada pursaetha DC. & Mimosaceae & WV & Str-UA \\
\hline 70 & Flagellaria indica $\mathrm{L}$. & Flagellariaceae & WV & TC \\
\hline 71 & Galactia longiflora Benth. & Papilionaceae & $\mathrm{HV}$ & ST \\
\hline 72 & Gloriosa superb L. & Liliaceae & $\mathrm{HV}$ & $\mathrm{RC}$ \\
\hline 73 & Gnetum ula Brongh & Gnetaceae & WV & Str-UA \\
\hline 74 & Gouania leptostachya DC. & Rhamnaceae & WV & $\mathrm{TC}$ \\
\hline 75 & Grewia rhamnifolia Roth. & Tiliaceae & WV & Str-UA \\
\hline 77 & Gymnopetalum cochinchinensis (Lour) Kurz. & Cucurbitaceae & WV & ST \\
\hline 78 & Hemidesmus indicus var. indicus (L.) R. Br. & Asclepiadaceae & $\mathrm{HV}$ & TC \\
\hline 79 & $\begin{array}{l}\text { Hemidesmus indicus var. pubescens (Wt. \& } \\
\text { Arn. Hook.f. }\end{array}$ & Asclepiadaceae & HV & ST \\
\hline 80 & Hewittia scandens (Milne) Mabb. & Convolvulaceae & WV & ST \\
\hline 81 & Hiptage benghalensis (L.) Kurz. & Malphigiaceae & WV & Str-A \\
\hline 82 & Holostemma ada-kodien Schult. & Asclepiadaceae & WV & Str-UA \\
\hline 83 & Hoya pendula Wt. \& Arn. & Asclepiadaceae & WV & ST \\
\hline 84 & Hugonia mystax $\mathrm{L}$. & Linaceae & WV & Str-UA \\
\hline 85 & Hypserpa nitida Miers & Menispermaceae & WV & $\mathrm{HC}$ \\
\hline 86 & Ichnocarpus frutescens (L.) Ait. & Apocynaceae & $\mathrm{HV}$ & ST \\
\hline 87 & Ipomoea eriocarpa R.Br. & Convolvulaceae & WV & ST \\
\hline 88 & Ipomoea hederifolia $\mathrm{L}$. & Convolvulaceae & $\mathrm{HV}$ & ST \\
\hline 89 & Ipomoea nil (L.) Roth & Convolvulaceae & $\mathrm{HV}$ & ST \\
\hline 90 & Ipomoea obscura (L.) Ker-Gawl.) & Convolvulaceae & $\mathrm{HV}$ & ST \\
\hline 91 & Ipomoea pes-tigridis $\mathrm{L}$. & Convolvulaceae & $\mathrm{HV}$ & ST \\
\hline 92 & Ipomoea sepiaria Roxb. & Convolvulaceae & WV & ST \\
\hline 93 & Ipomoea staphylina Roem. \& Schult. & Convolvulaceae & WV & ST \\
\hline 94 & Ipomoea turbinata Lag. & Convolvulaceae & WV & ST \\
\hline 95 & Ipomoea wightii (Wall.) Choisy & Convolvulaceae & WV & ST \\
\hline
\end{tabular}


63

\begin{tabular}{|c|c|c|c|c|}
\hline 96 & Jacquemontia paniculata (Brum.f.) Hallier.f. & Convolvulaceae & $\mathrm{HV}$ & ST \\
\hline 97 & Jasminum angustifolium Vahl & Oleaceae & WV & ST \\
\hline 98 & Jasminum arborescens Roxb. & Oleaceae & WV & ST \\
\hline 99 & Jasminum roxburghianum Wall. & Oleaceae & WV & ST \\
\hline 100 & Leptadenia reticulata (Retz.) Wt. \& Arn. & Asclepiadaceae & WV & ST \\
\hline 101 & Mallotus repandus Muell.-Arg. & Euphorbiaceae & WV & Str-A \\
\hline 102 & Merremia hederacea (Burm.f.) Hallier $\mathrm{f}$. & Convolvulaceae & $\mathrm{HV}$ & ST \\
\hline 103 & Merremia tridentate (L.) Hallier $\mathrm{f}$. & Convolvulaceae & $\mathrm{HV}$ & ST \\
\hline 104 & Merremia tridentata s.sp tridentata (Desr.) Oostr. & Convolvulaceae & $\mathrm{HV}$ & ST \\
\hline 105 & Merremia umbellata (L.)Hallier $\mathrm{f}$. & Convolvulaceae & $\mathrm{HV}$ & ST \\
\hline 106 & Merremia vitifolia (Burm.f.) Hallier f. & Convolvulaceae & WV & ST \\
\hline 107 & Millettia auriculata Brandis & Papilionaceae & WV & Str-UA \\
\hline 108 & Millettia racemosa (Wt. \& Arn. Benth. & Papilionaceae & WV & Str-UA \\
\hline 109 & Mimosa intsia $\mathrm{L}$. & Mimosaceae & WV & Str-A \\
\hline 110 & Momordica charantia L. & Cucurbitaceae & WV & TC \\
\hline 111 & Momordica dioica Willd. & Cucurbitaceae & WV & TC \\
\hline 112 & Morinda umbellata $\mathrm{L}$. & Rubiaceae & WV & Str-UA \\
\hline 113 & Mucuna gigantea DC. & Papilionaceae & WV & ST \\
\hline 114 & Mucuna monosperma Wt. & Papilionaceae & WV & ST \\
\hline 115 & Mucuna nigricans (Lour.) Steud. & Papilionaceae & WV & ST \\
\hline 116 & Mucuna pruriens (L.) DC. & Papilionaceae & WV & ST \\
\hline 117 & Mukia maderaspatana (L.) Roem. & Cucurbitaceae & WV & TC \\
\hline 118 & Naravalia zeylanica (L.) DC. & Rananculaceae & WV & $\mathrm{TC}$ \\
\hline 119 & Olax scandens Roxb. & Olacaceae & WV & Str-UA \\
\hline 120 & Operculina turpethum (L.) Silva Manso & Convolvulaceae & WV & ST \\
\hline 121 & Oplia amentacea Roxb. & Opiliaceae & WV & ST \\
\hline 122 & Paracalyx scariosus (Roxb.) Ali & Papilionaceae & WV & ST \\
\hline 123 & Passiflora foetida L. & Passifloraceae & $\mathrm{HV}$ & TC \\
\hline 124 & Pergularia daemia (Forssk.) Chiov. & Asclepiadaceae & $\mathrm{HV}$ & ST \\
\hline 125 & Pisonia aculeata $\mathrm{L}$. & Nyctaginaceae & WV & Str-A \\
\hline 126 & Plecospermum spinosum Trecul & Moraceae & WV & Str-A \\
\hline 127 & Pterolobium hexapetalum (Roth) Sant. \& Wagh & Caesalpiniaceae & WV & Str-A \\
\hline 128 & Pueraria tuberosa DC. & Papilionaceae & WV & Str-UA \\
\hline 129 & Reissantia indica (Willd.) Halle & Celastraceae & WV & Str-UA \\
\hline 130 & Rhaphidophora decursiva (Roxb.) Schott. & Convolvulaceae & WV & ST \\
\hline 131 & Rivea hypocrateriformis (Desr.) Choisy & Araceae & WV & $\mathrm{RC}$ \\
\hline 132 & Rubia cordifolia L. & Rubiaceae & $\mathrm{HV}$ & ST \\
\hline 133 & Rubus ellipticus Sm. & Rosaceae & WV & Str-A \\
\hline 134 & Rubus niveus Thunb & Rosaceae & WV & Str-A \\
\hline 135 & Salacia chinensis L. & Celastraceae & WV & Str-UA \\
\hline 136 & Sarcostemma acidum (Roxb.) Voigt & Asclepiadaceae & WV & Str-UA \\
\hline 137 & Schefflera roxburghii Gamble & Araliaceae & WV & Str-UA \\
\hline 138 & Schefflera stellata (Gaertn.) Harms & Araliaceae & WV & Str-UA \\
\hline 139 & Schefflera venulosa (Wt. \& Arn.) Harms & Araliaceae & WV & Str-UA \\
\hline 140 & Scindapus officinalis (Roxb.) Schott. & Araceae & WV & $\mathrm{RC}$ \\
\hline 141 & Scutia maritime (Burm.f.) Kurz. & Rhamnaceae & WV & Str-A \\
\hline 142 & Secamone emetic (Retz.) R.Br. & Asclepiadaceae & WV & ST \\
\hline 143 & Smilax zeylanica L. & Smilacaceae & $\mathrm{HV}$ & TC \\
\hline 144 & Solanum trilobatum $\mathrm{L}$. & Solanaceae & WV & Str-A \\
\hline 145 & Solena amplexicaulis (Lam.) Gandhi & Cucurbitaceae & WV & TC \\
\hline 146 & Stemona tuberosa Lour. & Stemonaceae & $\mathrm{HV}$ & $\mathrm{RC}$ \\
\hline 147 & Stephania japonica (Thunb.) Miers. & Menispermaceae & $\mathrm{HV}$ & ST \\
\hline 148 & Symphorema involucratum Roxb. & Verbenaceae & WV & ST \\
\hline 149 & Teramnus labialis (L.f.) Spreng. & Papilionaceae & WV & ST \\
\hline 150 & Teramnus mollis Benth. & Papilionaceae & WV & ST \\
\hline 151 & Thunbergis fragrans Roxb. & Acanthaceae & $\mathrm{HV}$ & ST \\
\hline
\end{tabular}




\begin{tabular}{|c|c|c|c|c|}
\hline 152 & Tiliocora acuminata (Lam.) Miers. & Menispermaceae & WV & ST \\
\hline 153 & $\begin{array}{c}\text { Tinospora cordifolia (Willd.) Hook.f. \& } \\
\text { Thoms. }\end{array}$ & Menispermaceae & WV & ST \\
\hline 154 & Tinospora sinensis (Lour.) Merr. & Menispermaceae & WV & ST \\
\hline 155 & Toddalia asiatica (L.) Lam. & Rutaceae & WV & $\mathrm{HC}$ \\
\hline 156 & Toxocarpus kleinii Wt. \& Arn. & Asclepiadaceae & WV & ST \\
\hline 157 & Tragia involucrata $\mathrm{L}$. & Euphorbiaceae & WV & ST \\
\hline 158 & Tragia plukenetii R.Sm. & Euphorbiaceae & $\mathrm{HV}$ & ST \\
\hline 159 & Trichosanthes cucumerina $\mathrm{L}$. & Cucurbitaceae & WV & TC \\
\hline 160 & Trichosanthes tricuspidata Lour. & Cucurbitaceae & WV & $\mathrm{TC}$ \\
\hline 161 & Tylophora fasciculata $\mathrm{Wt}$. & Asclepiadaceae & $\mathrm{HV}$ & ST \\
\hline 162 & Tylophora indica (Burm.f.) Merr. & Asclepiadaceae & HV & ST \\
\hline 163 & Tylophora macrantha Hook.f. & Asclepiadaceae & $\mathrm{HV}$ & ST \\
\hline 164 & Tylophora rotundifolia (Roxb.) Wt. \& Arn. & Asclepiadaceae & $\mathrm{HV}$ & ST \\
\hline 165 & Uncaria sessilifructus Roxb. & Rubiaceae & WV & $\mathrm{HC}$ \\
\hline 166 & Vanilla wightiana Lindl. & Orchidaceae & $\mathrm{HV}$ & $\mathrm{RC}$ \\
\hline 167 & Ventilago denticulata Willd. & Rhamnaceae & WV & ST \\
\hline 168 & Wattakaka volubilis (L.f.) Stapf & Asclepiadaceae & WV & ST \\
\hline 169 & Ziziphus oenoplia (L.) Mill. & Rhamnaceae & WV & Str-A \\
\hline 170 & Ziziphus rugosa Lam. & Rhamnaceae & WV & Str-A \\
\hline
\end{tabular}

WV- Woody vines; HV-Herbaceous vines; ST-Stem twiners; STr-A-Straggler armed; STr-UA- Straggler unarmed; TC- Tendril climber; RC-Root climbers; HCHook climbers

\section{Conclusion}

Lianas play a key role in the ecology and dynamics of forests and may be helpful in conservation of forest resources. The present study has shown that the tropical forests of northern Eastern Ghats harbor has a high floristic diversity of lianas, which contribute to the overall biodiversity of the forests. These forests are deteriorating under constant anthropogenic activities. The present data of floristic diversity of lianas would be useful in species conservation and management. The importance of climbers can be useful to biologists in the establishment of a standardized methodology and to provide these data on the structural threats to tropical forests for a global audience.

\section{Acknowledgements}

We are thankful to the Department of Biotechnology, Government of India for the financial support and to Andhra Pradesh Forest Department for their co-operation during field work.

\section{References}

Acevedo-Rodriguez P (2005). Vines and climbing plants of Puerto Rico and the Virgin Islands Contribution from the United States National Herbarium 51:1-483.

Araujo D, Alves M (2010). Climbing plants of a fragmented area of lowland Atlantic Forest, Igarassu, Pernambuco (northeastern Brazil). Phytotaxa 8:1-24.

Burnham RJ (2004). Alpha and beta diversity and distribution of lianas in Yasuni, Equador. Forest Ecol Manag 190:4355.
Champion HG, Seth SK (1968). The revised forest types of India. Government of India, New Delhi.

Gamble JS, Fischer CEC (1915-1935). Flora of the Presidency of Madras. London: Adlard \& son. Vols. I-III.

Gentry AH (1991). The distribution and evaluation of climbing plants: p. 3-49. In: Putz FE, Mooney HA (Eds.). The biology of vines, Cambridge University Press, Cambridge, U.K.

Gollasimood S, Faridah-Hanum I, Nazre M, Kamziah AK (2012). Abundance and distribution of climbers in a coastal hills forest in Perak, Malaysia. Journal of Agricultural Science 4(5):245-254.

Hegarty, EE, Caballe G (1991). Distribution and abundance in forests: p. 313-335. In: Putz FE, Mooney HA (Eds.). The biology of vines, Cambridge University Press, Cambridge, U.K.

Heijden GMF, Phillips OL (2008). What controls liana success in neotropical forests? Global Ecol Biogegr 17:372378.

Ingwell LL, Wright SJ, Becklund KK, Hebbell SP, Schnitzer SA (2010). The impact of lianas on 10 years of tree growth and mortality on Barro Colorado Island, Panama. J Ecol 98:879-887.

Kuzee ME, Bongers F (2005). Climber abundance, diversity and colonization in degraded forests of different ages in Côte d'Ivoire: p. 67-84. In: Bongers F, Parren MPE, Traore D. (Eds.). Forest climbers of West Asia: Diversity, Ecology and Management. CABI Publishing.

Londre RA, Schnitzer SA (2006). The distribution of lianas and their change in abundance in temperate forests over the past 45 years. Ecology 87:2973-2978. 
65

Muthumperumal C, Parthasarathy N (2009). Angiosperms, climbing plants in tropical forests of southern Eastern Ghats, Tamil Nadu, India. Checklist 5(1):092-111.

Nabe-Nielsen J (2001). Diversity and distribution of lianas in a neotropical rainforest, Yasuni National Park, Ecuador. J Trop Ecol 17:1-19.

Parthasarathy N, Muthuramkumar S, Reddy MS (2004). Patterns of liana diversity intropical evergreen forests of peninsular India. Forest Ecol Manag 190:15-31.

Phillips OL, Martinez RV, Arroyo L, Baker TR, Killeen T, Lewis SL, Malhi Y, Mendosa AM, Neill D, Vargas PN, Alexiades M, Ceron C, Fiore AD, Erwin T, Jardim A, Palacios W, Saldias M, Vinceti B (2002). Increasing dominance of large lianas in Amazonian forests. Nature 418:770-774.

Putz FE, Mooney HA (1991). The Biology of vines, p. 53-72 In Cambridge University Press, Cambridge, United Kingdom.

Rao GVS, Kumari GR (2002-2008). Flora of Visakhapatnam district. Andhra Pradesh. Vol. I \&II. Botanical Survey of India. Kolkata.

Reddy MS, Parthasarathy N (2006). Liana diversity and distribution on host trees in four inland tropical dry evergreen forests of peninsular India. Trop Ecol 47(1):109123.

Reddy MS, Parthasarathy N (2003). Liana diversity and distribution in four tropical dry evergreen forests on the Coromandel coast of south India. Biodivers Conserv 12:1609-1627.
Sarvalingam A, Rajendran A (2012). Diversity of liana species in Maruthamalai hills of southern Western Ghats, India. Phytotaxonomy 12:131-135.

Schnitzer SA, Carson WP (2010). Lianas suppress tree regeneration and diversity in tree fall gaps. Ecol lett 13:849857.

Schnitzer SA, Kuzee ME, Bongers F (2005). Disentagling above-and below-ground competition between lianas and trees in a tropical forest. J. Ecol 93:1115-1125.

Schnitzer SA, Bongers F (2002). The ecology of lianas and their role in forests. Trends in Ecology and Evolution 17(5):223-230.

Schnitzer SA, Dalling JW, Carson WP (2000). The impact of lianas on tree generation in tropical forest canopy gaps: Evidence for an alternative pathway of gap-phase regeneration. J Ecol 88:655-666.

Yuan C, Liu W, Tang CQ, Li XS (2009). Species composition, diversity and abundance if lianas in different secondary and primary forests in a subtropical mountainous area, SW China. Ecol Res 24:1361-1370.

Wright SJ, Jaramillo AM, Pavon J, Condit R, Hubbell SP, Foster RB (2005). Reproductive size thresholds in tropical trees: variation among individuals, species and forests. J Trop Ecol 21:307-3115.

Zotz G, Cueni N, Korner C (2006). In-situ growth stimulation of a temperate zone liana (Hedera helix) in elevated $\mathrm{CO}_{2}$. Functional Ecology 20:763-769. 